http://bjas.journals.ekb.eg

\title{
Expert Tibial Intramedullary Nailing For proximal and Distal Tibial Fractures
}

M.Mashhour, E.M.Bayomy, S.A.Shoulah, M. E.Elkady

Orthopedic Surgery Dept., Faculty of Medicine, Benha Univ., Benha, Egypt

E-Mail: Elkady26@gmail.com

\begin{abstract}
Fractures of the proximal or distal thirds of the tibia are one of the most challenging injuries in orthopedic. A few strategies have been portrayed $\mathrm{m}$ the decision of procedure relies upon numerous elements including crack arrangement, bone quality, delicate tissue injury and accessible equipment.Intramedullary nailing might be powerful in dealing with these breaks even with straightforward articular augmentation, yet care should be taken with decrease and sufficient screws locking to forestall malalignment and ceaseless knee agony may likewise be an issue. This investigation included 20 patients with distal and proximal tibial breaks rewarded with intramedullary nail with multidirectional proximal and distal locking screws (Expert tibial nail). The time of follow up was up to 6months utilizing certain scoring frameworks. The suggestive and useful assessment results were reviewed by the models by Johner and Wruh's Criteria According to Johner and Wruh's measures, $45.0 \%$ of patients indicated great rules, $30.0 \%$ were brilliant, $15.0 \%$ were reasonable and just $10.0 \%$ were poor. Two cases created deferred association (10\%), two cases built up Sudeck's decay (10\%), one case created malunion (5\%), one case created nonunion( $5 \%$ ) and foremost knee torment was found in four cases $(20 \%)$. So front knee torment spoke to the most widely recognized intricacy in this study.Treatment of proximal and distal thirds tibial cracks utilizing master tibial intramedullary nail (with multidirectional locking screws) is a protected and acknowledged strategy option in contrast to customary nail and plating procedure. This structure of the embed helps in accomplishing stable obsession and improved securing most extraarticular tibial cracks.
\end{abstract}

\section{Introduction}

The tibia is as of now the most ordinarily cracked long bone in the body with a yearly occurrence of two tibial shaft breaks for each 1000 people. The normal period of patients with tibial shaft cracks is around 37 years, and adolescent guys are accounted for to have the most elevated frequency [1]) .

there are numerous ways that an individual can endure a tibia crack. These incorporate awful wounds, for example, engine vehicle mishaps or falls ,sports that include rehashed effect on the shin, for example, significant distance running, wounds from physical games, for example, football [2].

Various treatment alternatives exist for rewarding tibial breaks and great outcomes have been accounted for with both moderate and careful strategies. To diminish the intricacies asssociated with preservationist treatment, propensity towards employable administration of tibial breaks become progressively famous. Different employable techniques like open decrease and plating, intramedullary nailing and outer obsession have their own signs, favorable circumstances and burdens [3].

Interlocking nail has extended the scope of signs for medullary osteosynthesis of tibial shaft cracks to incorporate pretty much every kind of break .Intramedullary nailing offers an alluring treatment, stable obsession with early rebuilding of capacity without utilization of mortar and evade the difficulties related with plating as ulceration of skin overlying plate, profound disease prompting osteomyelitis and refracture and postponed association. Anyway there are a few issues in treatment of break tibia with traditional intramedullary interlocking nailing like trouble in controlling cracks of proximal and distal 1/third tibia and communited metaphyseal cracks $[4,5]$.

These weaknesses of traditional intramedullary interlocking nail in overseeing proximal and distal third cracks have been overwhelmed by the presentation of Expert Tibial Interlocking Nail because of adjustments in usable methods, its structure and headway in locking screws.Thus master tibial nail configuration permits better control in metaphyseal tibial fragments through various interlocking openings in closeness to either end of the nail. Multidirectional interlocking screws guarantee that arrangement can be all around kept up and solidness safeguarded regardless of a short proximal or distal piece [6].

The Expert Tbial Nail System (ETNS notwithstanding the standard static and dynamic locking choices, the ETNS highlights multi directional securing alternatives in the distal and proximal piece of the nail, 5 proximal locking options(three novel angled and two medio-sidelong bolting choices) and 4 distal locking options(Two medio-parallel ,one antero-back and one extra diagonal locking choice, put distally) .. End top square the most proximal screw making a precise stable build $[7,8]$.

The utilization of multidirectional interlocking screws guarantees that arrangement can be kept up and that dependability can be protected in spite of a short proximal or distal tibial section [9].

Postoperative confusions of intramedullary ETN obsession of tibia breaks incorporate postponed association, essential and optional malalignment, embed related complexities, and auxiliary medical procedure yet with lower rates in contrast with ordinary intramedullary interlocking nail [10]. 
The point of this investigation was to assess the result of Expert Tbial Nail System (ETNS) in obsession of proximal and distal extra articular tibial fructures.

\section{Patient and method}

This investigation included 20 patients (14 guys and 6 females) with distal and proximal tibial fractures(4 proximal and 16 distal ) rewarded between January 2019 and January 2020 at Banha University Hospital with intramedullary nail with multidirectional proximal and distal locking screws (Expert tibial nail). Their ages went from 20 years to 59 years with a normal of 35 years. The time of follow up was as long as a half year.

On confirmation, all patients were exposed to history taking, clinical assessment, radiological and research center examinations. Full guiding of members in this exploration and educated assent was acquired with full Privacy of members and secrecy of the information.

Consideration Criteria was (Extraarticular metaphyseal breaks of proximal and distal tibia (straightforward, wedge or complex), Skeletally develop understanding, Primary mediation, Coemployable restoratively sound patient and Open fructures,

Rejection Criteria was (Intra-articular cracks, open breaks grade III with tainting and, Patient with dynamic contamination)

All the patients were surveyed at the hour of last follow up utilizing certain scoring systems. The suggestive and utilitarian assessment results were reviewed by the measures by Johner and Wruh's Criteria (105). Clinical outcomes were reviewed as astounding, great, reasonable, or poor .

The planning of medical procedure was enhanced to permit the delicate tissues to settle and limit the postoperative injury issues frequently connected with the careful administration of these intricate cracks.

Within the sight of extreme delicate tissue growing or skin rankles, the medical procedure was deferred for about seven days until the expanding has died down and the skin starts to wrinkle.

Postoperative evaluation of general condition and lower appendage for the initial 24 hours Plain $\mathrm{X}$-beam was done (A.P. furthermore, sidelong perspectives) after the activity for the evaluation of crack decrease. Quick postoperative rise of the appendage for in any event 2 or 3 days, dynamic and detached scope of movement of knee were begun IV anti-infection inclusion proceeded for 5 days postoperatively, contingent upon the state of the injury. Wound consideration and dressing 48 hours after medical procedure.

The patients were released with guidelines to begin knee practice with opportunities for contact weight bearing for about a month and a half. In cases with fixed fibular cracks a beneath knee non- weight bearing cast was accomplished for about a month and a half before starting scope of movement exercises.

Fastens were evacuated 10 to 14 days postusable. Follow up visits were at two, six and 12 weeks postoperative in comminuted temperamental cases follow up visits were each ( 2 weeks). Clincal follow up evaluated for agony, delicacy and portability of crack site

Raiographic follow up of the patients evaluated for upkeep of decrease, appendage arrangement, refoundation of bone progression, slackening of metal and callus development. Radiographic association was characterized as the nearness of spanning callus in three of the four cortices as observed on foremost back and horizontal radiographs.

Weight-bearing was prohibited until about a month and a half after medical procedure, the patients were permitted to non-weight bearing ambulation with props. Incomplete weight-bearing strolling was permitted 6 to about two months postoperatively when proof of callus spanning was seen on plain radiographs.

By 3 months the patient can be progressed to full weight - bearing relying upon radiological proof of mending. For seriously comminuted cracks and breaks indicating deferred mending, a more drawn out time of halfway weight bearing is recommended.

Starting administration was normally coordinated toward general patient adjustment. Crack bracing with above knee section and screening radiography was done from the outset. Individual data, any related clinical issue as Diabetes Mellitus or propensities as smoking, the kind of break, time and instrument of injury were likewise reported.

The skin and delicate tissue around the break were deliberately inspected for scraped spot, wounds, injury and cut that may defer open techniques or meddle with the utilization of inside obsession. The anteroposterior and horizontal perspectives on tibia were finished. CT assessment was done in certain patients for identification of intra-articular breaks. The patient got antiedematous, analgesics and anti-infection agents in instances of extreme delicate tissue harm for counteraction of contamination.

\section{Results}

Mean age of the study population was 35 years with standard deviation of 14 years. As regard gender, $70.0 \%$ of study population were males with only $30 \%$ females. $20.0 \%$ were smokers and $15.0 \%$ had diabetes.

The most frequent mechanism of trauma was RTA $(80.0 \%)$ followed by falling down and fall from height ( $10.0 \%$ for each) $95.0 \%$ of patients showed associated fibular fractures and $15.0 \%$ showed associated other fractures. $45.0 \%$ of study 
population showed right side affection while $55.0 \%$ showed left side affection.

The most frequent time of fiation was $3-7$ days $(45.0 \%)$ followed by $1-3$ days $(30.0 \%)$ then
7 - 10 day (20.0\%) while the least frequent was 10 -14 days (5.0\%) Fig (1).



Fig ( 1) final functional results

According to Johner and Wruh's criteria $45.0 \%$ of patients showed good criteria, $30.0 \%$ were excellent, $15.0 \%$ were fair and only $10.0 \%$ were poor. The excellent and good results were considered as satisfactory results while the unsatisfactory included the fair and the poor results. Thus, satisfactory results were found in 15 patients $(75 \%)$, and the unsatisfactory results were found in 5 patients (25\%) Fig (2) .

Mean time of union was 16 weeks with standard deviation of 6 weeks. There was no correlation between time of union and age $(r=$ $0.408 \& \mathrm{P}$ value $=0.074$ )

Time of union was significantly higher in diabetics (20 week) compared to non-diabetics (16 week). $\mathrm{P}$ value was 0.04 . There were no significant differences in time of union as regrad gender, smoking status, side and site of fracture.
One case developed nonunion and further intervention had been done (bone graft and additional plate fixation). It represented $5 \%$ of all cases. Two cases developed delayed union. Union started to appear after 20 weeks and completed at 24 weeks. It represented $10 \%$ of all cases.

Four cases developed anterior knee pain. It was mainly due to the incision scar and infrapatellar pad scar. There was no need for continous medical treatment.It represented $20 \%$ of all cases. One case developed malunion due to malaligment after fixation. It represented 5\% of all cases.

Two cases developed Sudeck's atrophy. One case was 35 years old smoker male. And the other was diabetic female patient 55 years old. They received treatment in the form of calcitonin and pain killer and they showed improvement after physiotherapy. It represented $10 \%$ of all cases Table (1,2 and 3), Fig (2,3).

Table (1) Time Of Union As Regard Patients Charachteristics.

\begin{tabular}{lcccc}
\hline & & \multicolumn{2}{c}{ Time of union (Wks) } & \multirow{2}{*}{ P value } \\
\hline Sex & Mean & SD & 0.274 \\
\multirow{2}{*}{ Smoking } & Memales & 16 & 6 & \multirow{2}{*}{0.29} \\
\multirow{3}{*}{ DM } & Non-smoker & 17 & 4 & \\
& Smoker & 21 & 3 & 0.04 \\
Side & Non-diabetic & 16 & 6 & \multirow{2}{*}{0.882} \\
\multirow{2}{*}{ site of fracture } & Diabetic & 20 & 4 & \multirow{2}{*}{0.148} \\
& Right & 17 & 7 & \\
\hline
\end{tabular}

Mann Whitney U test was used 


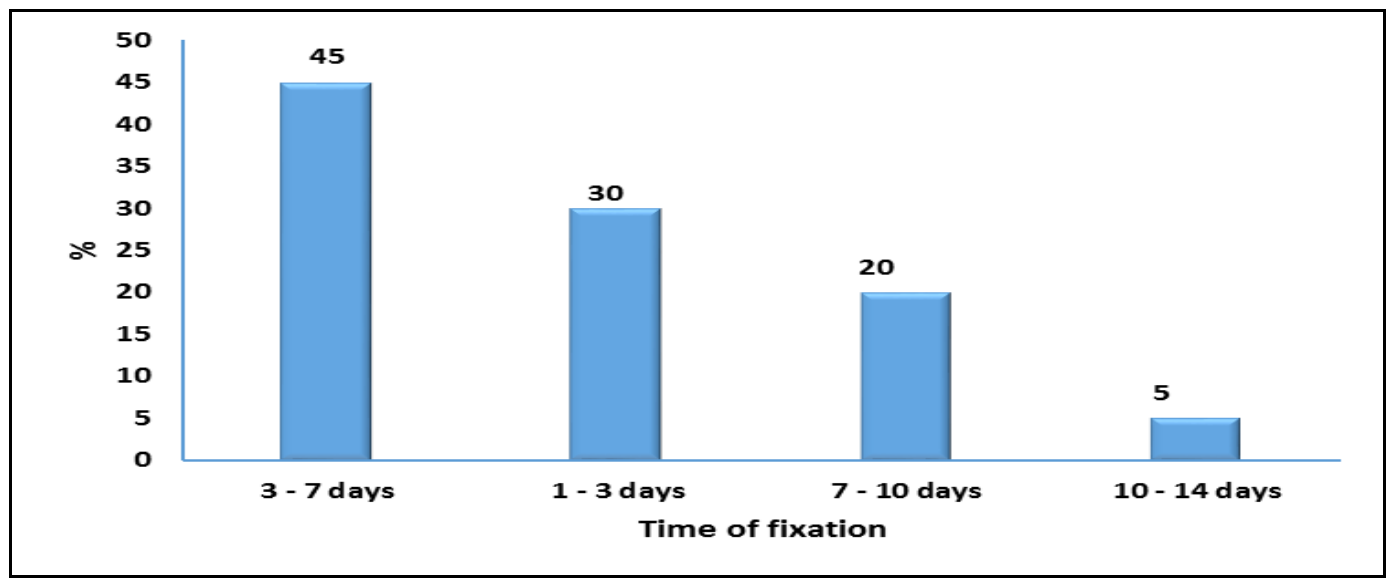

Fig (2) Time Of Fixation.

Table (2) Associated fibular and other fractures.

\begin{tabular}{|c|c|c|}
\hline & & n (\%) \\
\hline Associated fibular fracture & Yes & $19(95.0)$ \\
\hline Associated other fractures & Yes & $3(15.0)$ \\
\hline \multirow{2}{*}{$\begin{array}{l}\text { Type of associated } \\
\text { fractures }\end{array}$} & Neck femur \& tibia on other side & $1(33.3)$ \\
\hline & $\begin{array}{l}\text { Shaft femur ipsilat \& tibia contralat } \\
\text { Shaft humerus ipsilat \& tibia contralat }\end{array}$ & $\begin{array}{l}1(33.3) \\
1(33.0)\end{array}$ \\
\hline
\end{tabular}

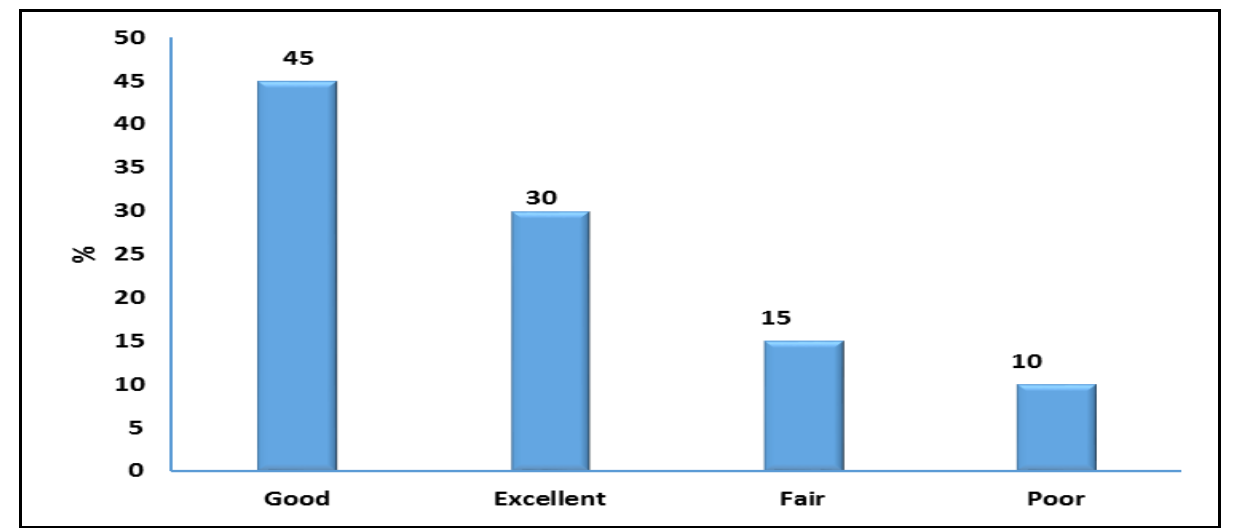

Fig ( 3) final functional results.

Table ( 3) Time Of Union As Regard Patients Charachteristics.

\begin{tabular}{lcccc}
\hline & \multicolumn{2}{c}{ Time of union (Wks) } & \multirow{2}{*}{ P value } \\
\cline { 2 - 3 } Sex & & Mean & SD & 0.274 \\
\multirow{2}{*}{ Smoking } & Males & 16 & 6 & \multirow{2}{*}{0.29} \\
\multirow{3}{*}{ DM } & Females & 17 & 4 & \\
& Non-smoker & 15 & 3 & 0.04 \\
Side & Smoker & 21 & 11 & \multirow{2}{*}{0.882} \\
\multirow{2}{*}{ site of fracture } & Non-diabetic & 16 & 6 & \multirow{2}{*}{0.148} \\
& Diabetic & 20 & 4 & 7 \\
\hline
\end{tabular}

Mann Whitney U test was used. 


\section{Discussion}

The tibia is as of now the most normally cracked long bone in the body with a yearly occurrence of two tibial shaft breaks for every 1000 people. The normal time of patients with tibial shaft breaks is roughly 37 years, and adolescent guys are accounted for to have the most elevated occurrence.

Various treatment alternatives exist for rewarding tibial cracks and great outcomes have been accounted for with both moderate and careful techniques. To lessen the confusions asssociated with traditionalist treatment, propensity towards employable administration of tibial breaks become progressively well known. Different employable techniques like open decrease and plating, intramedullary nailing and outside obsession have their own signs, focal points and detriments.

The targets in the treatment in these cracks are quick and perfect recuperating, minimization of loss of capacity and counteraction of any deformity [11].

Any strategy for treatment must fulfill certain standards. Treatment ought to be basic, cheap, accessible in each middle managing injury, negligibly intrusive to ensure the vascularity of distal tibia with strong obsession and ought to be effective [12].

This investigation included 20 patients (14 guys and 6 females) with distal and proximal tibial fractures(4 proximal and 16 distal ) rewarded between January 2019 and January 2020 at Banha University Hospital with intramedullary nail with multidirectional proximal and distal locking screws (Expert tibial nail). Their ages extended from 20 years to 59 years with a normal of 35 years. The time of follow up was as long as a half year.

These patients were surveyed by Johner and Wruh's measures. Also, Clinical outcomes were evaluated as superb, great, reasonable, or poor as follows:

$45.0 \%$ of patients indicated great measures, $30.0 \%$ were brilliant, $15.0 \%$ were reasonable and just $10.0 \%$ were poor.. The magnificent and great outcomes were considered as acceptable outcomes while the inadmissible incorporated the reasonable and the helpless outcomes. Therefore, palatable outcomes were found in 15 patients $(75 \%)$, and the inadmissible outcomes were found in 5 patients (25\%). In this examination, the time of follow up ran from 3 to a half year with a normal of 4.5 months.

Contrasted with the examination done by Prashant B Kenganal et al ; The investigation included 30 affirmed instances of metaphyseal and diaphyseal breaks of tibia. All cases were treated with intramedullary obsession with "Master Tibial Interlocking Nail".Functional results were evaluated by the standards by Johner and Wruh's Criteria. $43.33 \%$ of patients accomplished incredible outcomes, $33.33 \%$ of patients accomplished great outcomes, $16.67 \%$ reasonable outcomes were seen and in two patients, the practical outcomes were poor $(6.67 \%)(13)$.

Contrasted with the investigation done by $\mathrm{N}$. D. Kachhap et al; The investigation included 30 patients with 30 cracks of tibia 26 patient were having break in distal $1 /$ third tibia and 4 patient were having crack of proximal 1/third tibia. Based on medical clinic for extraordinary medical procedure (HSS) knee score13 (43\%) patients had brilliant outcomes; $9(30 \%)$ had great outcomes, 05 $(17 \%)$ had reasonable outcomes and $3(10 \%)$ had poor result [14].

Contrasted with the investigation was finished by Nadeem Ali et al ;sixty patients with extra articular distal tibial cracks were partitioned into 2 gatherings, the primary gathering included 30 patients rewarded by distal tibial locking plating gathering (MIPO) and the subsequent gathering included 30 patients rewarded by shut intramedullary interlocking nailing. In the principal gathering, 21 patients $(70 \%)$ had acceptable outcomes, 8 patients $(26.6 \%)$ had reasonable outcomes and one patient had helpless outcomes while in the subsequent gathering, 19 patients $(63.3 \%)$ had palatable outcomes, 9 patients $(30 \%)$ had reasonable outcomes and 2 patients had poor results [15].

Time of association was fundamentally higher in diabetics (multi week) contrasted with nondiabetics (multi week). The most elevated frequency of confusions was in the diabetic patients. Two diabetic patients was muddled with Sudeck's decay.

There were no huge contrasts in time of association as regrad sex, smoking status, side and site of crack.

Mean time of association was four months with standard deviation of about a month and a half. This coordinated with the investigation was finished by Neetin P. Mahajan et al; 46 patients with distal tibial metadiaphyseal breaks were treated by Expert nail tibia, The normal time of association was 16.4 weeks (ran from 12 to 28 weeks) (16).it likewise coordinated with the examination was finished by Ramachandra et al as the normal time of association was four months of 72 patients with distal tibial cracks rewarded by master nail tibia [17]. Comparative outcomes detailed by Kumar YC et al as the normal time of association was four months for nailing (Expert nail) gathering and for plating bunch it was 18 weeks [18].

Anyway more slow paces of association was accounted for Prashant B Kenganal et al; as the normal time of association was 21.04 weeks(108).

In examination with MIPPO, Guo et al ;revealed no critical contrasts among nailing and MIPPO regarding time to association, however nailing indicated lesser activity and fluoroscopy use times, and better capacity and alignment [19]. 
Age extended from 20 years to 59 years with a normal of 35 years. Age was found to have measurably irrelevant impact at long last aftereffects of this investigation yet had factually critical impact in the hour of association. The most noteworthy occurrence of palatable outcomes happened at the age of 20-30 years of age. These outcomes were about the equivalent with that detailed by Mohit Bihani et al; in his investigation of intramedullary obsession of distal tibial breaks around diametaphysis utilizing Expert tibial nail, who found that age had noteworthy impact at long last results(115).

In this investigation, two cases created postponed association (10\%), two cases built up Sudeck's decay (10\%), one case created malunion(5\%), one case created nonunion(5\%) and front knee torment was found in four cases(20\%). So front knee torment spoke to the most widely recognized inconvenience in this investigation.

Mohit Bihani et al ;in their examination, additionally announced that commonest complexity was foremost knee torment (42\%) [20].

The moderately low number of patients, short follow up period and the nonattendance of control bunch are constraints to this investigation.

All in all, this examination accentuated the clinical achievement and low horribleness related with the utilization of intramedullary nail tibia with multidirecrtional distal locking screws (master nail). This was fit to the administration of proximal and distal tibial breaks. Diminished occurrence of delicate tissue entanglements, early weight bearing and great practical recuperation, these contrast well and other revealed medicines for patients with proximal or distal tibial cracks and prove the suggestions for master nail ought to be the treatment of decision for such wounds.

\section{References}

[1] D.Wennergren, C.Bergdahl, J.Ekelund. Epidemiology and incidence of tibia fractures in the Swedish Fracture Register. Injury, Vol. Sep 7,2018.

[2] Tibia (shinbone) shaft fractures. Retrieved from

https://orthoinfo.aaos.org/en/diseases-conditions/tibia-shinbone-shaftfractures, 2010 .

[3] Larsen, Peter. "Incidence and epidemiology of tibial shaft fractures." Injury, Vol. 46.4 , PP.746-750,2015.

[4] S.Kuhn, M.Hansen, P.M.Rommers. "Extending the Indications of Intramedullary Nailing with the Expert Tibial Nail@." Acta chirurgiae orthopaedicae et traumatologiae Cechoslovaca, Vol. 75, PP.2-77,2008.

[5] Chaudhari, Kartavya. "A Comparative Study of Surgical Management of Distal
Tibial Fractures Treated with Locking Compression Plate by Minimally Invasive Percutaneous Plate Osteosynthesis (MIPPO) and Expert Tibial Nail." EC Orthopaedics, Vol. 9, PP. 702-714, 2018.

[6] Pandey, Anil. "Comparative Analysis Of Closed Reduction And Expert Tibia Nailing With Open Reduction And Plate \& Screw Fixation In The Treatment Of Proximal Tibia Extraarticular Fractures." International J.,Medical and Biomedical Studies ,2018.

[7] D.C.Zhua, L.Liub, F.Gaoa. Comparison of closed reduction and expert tibial nailing with open reduction and plate and screw fixation in the treatment of two segmental tibial fractures. Chinese J Traumatol, Vol.18(4), PP.219-22,2015.

[8] Attal, Rene. "A multicentre case series of tibia fractures treated with the Expert Tibia Nail (ETN)." Archives of orthopaedic and trauma surgery , Vol.132.7, PP.975984,2012.

[9] RATHOR, NAVEEN. "Management of Distal Tibia Metadiaphyseal FractureMinimal Invasive Percutaneous Plate Osteosynthesis (MIPPO) By Medial LCP Versus Expert Tibia Nailing (ETN): A Comparative Study." Indian J.,Applied Research, Vol.9, PP. 7.3 ,2018.

[10]Li, Yong. "Comparison of low, multidirectional locked nailing and plating in the treatment of distal tibial metadiaphyseal fractures." International orthopaedics, Vol. 36.7, PP.14571462,2012.

[11]D.J.Redfern, S.U.Syed, S.J.Davies. Fractures of the distal tibia, minimally invasive plate osteosynthesis. Injury, Vol.35, PP.615-20,2004.

[12] Coles CP, Gross M .Closed tibial shaft fractures, management, treatment and complications a review of the prospective literature .Can. J. surg. 2000; 43: 226-56.

[13] Kenganal, B.Prashant. "Functional outcome of metaphyseal and diaphyseal fractures of tibia treated with expert tibial interlocking nail: A prospective study." International J.,Orthopaedics, Vol. 5.1, PP.48-52,2019.

[14] N.D.Kachhap. "To study outcome of surgical management of distal 3rd and proximal 3rd fractures of tibia in adults using expert tibial nails." International J.,Research in Orthopaedics, Vol. 3.4, PP. 862. ,2017.

[15] Nadeem Ali, Abedullah Bhat, Firdous Ahmed Bangroo. Treatment of extra articular distal tibial fractures: minimally invasive percutaneous plate osteosynthesis 
versus intramedullary nailing. Trauma Mon. July, Vol. 22(4), PP.e19593,2017.

[16] N.p.Mahajan, E.D.Pawar, A.C.Supe. Role of tibia interlocking nail in treating distal tibial metadiaphyseal fractures: study of 46 cases. International J.,Research in Orthopedics, Vol.4, PP.65-71, 2018.

[17] N.Ramachandra, M.Badami1, M.Shantharam. Management of distal tibial metaphyseal fractures by expert tibial nail Indian J.,Orthopaedics Surgery, Vol.3(3), PP.229-33, 2017.

[18]C.Yashavantha Kumar, M.S.Shivaprased, V.Trilok. treatment of distal tibial fractures: plating versus intramedullary nailing. International J.,Research in Orthopedics, Vol. 2, PP.116-9,2016.
[19] J.J.Guo, N.Tang, H.L.Yang. A prospective, randomised trial comparing closed intramedullary nailing with percutaneous plating in the treatment of distal metaphyseal fractures of the tibia. $\mathrm{J}$ Bone Joint Surg Br, Vol. 92, PP.9848,2010.

[20] Mohit Bihani, P.Krishna Sravanth, Shardaindu Sharma. intramedullary fixation of distal tibial fractures around diametaphysis using locked intramedullary cannulated distal tibial nail aprospective study.International J.,Orthopedics sciences, Vol.2, PP.38-42,2016. 